\title{
Controlling the Cooperativity in the Supramolecular Polymerization of Ionic Discotic Amphiphiles via Electrostatic Screening
}

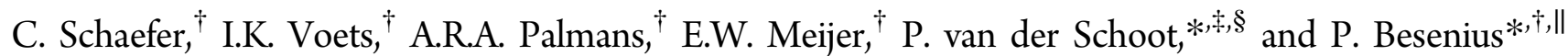

${ }^{\dagger}$ Institute for Complex Molecular Systems and Laboratory of Macromolecular and Organic Chemistry and Theory of Polymers and Soft Matter Group, Eindhoven University of Technology, P.O. Box 513, 5600 MB Eindhoven, The Netherlands

${ }^{\S}$ Institute for Theoretical Physics, University of Utrecht, Leuvenlaan 4, 3584 CE Utrecht, The Netherlands

"Organic Chemistry Institute and CeNTech, Westfälische Wilhelms-Universität Münster, Corrensstrasse 40, D-48149 Münster, Germany

\section{Supporting Information}

ABSTRACT: In a combined experimental and theoretical approach, we investigate the supramolecular polymerization of ionic discotic amphiphiles into nanorods of varying mean length, depending on the temperature and ionic strength of the buffered aqueous solution. Invoking a nucleated supramolecular polymerization model that explicitly deals with the effects of screened Coulomb interactions, we correlate the degree of cooperativity of the supramolecular polymerization with the ionic strength of the solution, as probed by means of circular dichroism spectroscopy. Experiment and theory show that electrostatic interactions between the amphiphiles in the rods make the polymerization less

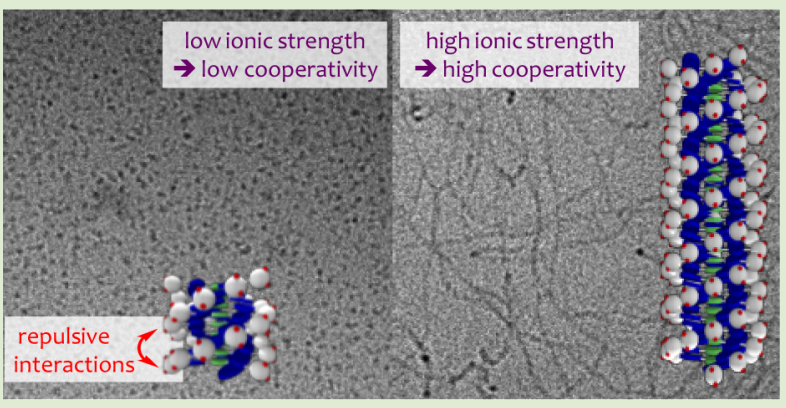
cooperative, implying that the larger the concentration of mobile ions in the solution the larger the cooperativity due to their screening effect. We furthermore extract quantitative information about the effective surface charge densities of the supramolecular nanorods in solution, a parameter that has been particularly difficult to determine experimentally in other related self-assembled systems.

$\mathrm{T}$ he phenomenon of cooperativity involves the collective or concerted behavior of many-particle or many-actor systems and presents itself in a myriad of different ways in chemistry, physics, and biology. It is of relevance not only in the context of macroscopic phase transitions and percolation phenomena, ${ }^{1}$ but expresses itself also in synergistic effects in organic catalysis, ${ }^{2}$ in nonadditive effects in solvation dynamics, ${ }^{3}$ and in allosteric and chelate effects in molecular recognition and in self-assembled macromolecules, including viruses and actin and tubulin filaments. ${ }^{4,5}$

A better understanding of cooperativity and the way it comes about is of key importance to obtain a comprehensive mechanistic understanding of supramolecular polymerizations, and may result in the ability to direct morphological and thermodynamic properties of self-assembling systems. ${ }^{6}$ Despite the current insights into the sources of cooperative selfassembly, the main ones being conformational switching and energetically unfavorable nucleating intermediates, ${ }^{7}$ active control over the degree of cooperativity, for example, through the rational design of the molecular building blocks, remains elusive. $^{8}$

An exception is a new class of ionic discotic amphiphiles based on a benzene-1,3,5-tricarboxamide (BTA) core, coupled to peripheral negatively charged Gd(III)-DTPA (DTPA = diethylenetriaminepentaacetic acid) via a perfluorinated phenylalanine linker that we, for brevity, refer to as the $C_{3}$-symmetric discotic amphiphile or $\mathrm{C}_{3} \mathrm{DA}$ (Figure 1). We recently found that $\mathrm{C}_{3} \mathrm{DAs}$ self-assemble in aqueous solution into onedimensional, nanometer-sized structures with a mean length that depends sensitively on the temperature, the concentration and the ionic strength of the (buffered) aqueous solvent. In fact, screening interactions between the charges on $\mathrm{C}_{3} \mathrm{DA}$ by the addition of $\mathrm{NaCl}$ to the solution induces a transition from short objects into elongated, rod-like assemblies. ${ }^{8 \mathrm{~d}}$ Superficially, this is not dissimilar to what has been seen in worm-like micellar solutions of charged surfactants. ${ }^{9}$ However, we have discovered that for $\mathrm{C}_{3} \mathrm{DA}$, Coulomb interactions not only impact upon the elongation of $\mathrm{C}_{3} \mathrm{DA}$ assemblies but also their nucleation from the monomeric to the aggregated state. This seems less pronounced in conventional surfactant systems, presumably because for these the cooperativity is dominated by energetically unfavorable assemblies with aggregation numbers below that of the smallest viable (spherical) micelle. ${ }^{9}$

Our previous investigations seemed to suggest that an increase in salt concentration screens the repulsive interactions between charged monomers, which induces a switch in the selfassembly mechanism. ${ }^{8 \mathrm{~d}, \mathrm{f}}$ We present here results of a systematic investigation on the impact of ionic strength on the activation

Received: May 2, 2012

Accepted: June 12, 2012

Published: June 15, 2012 


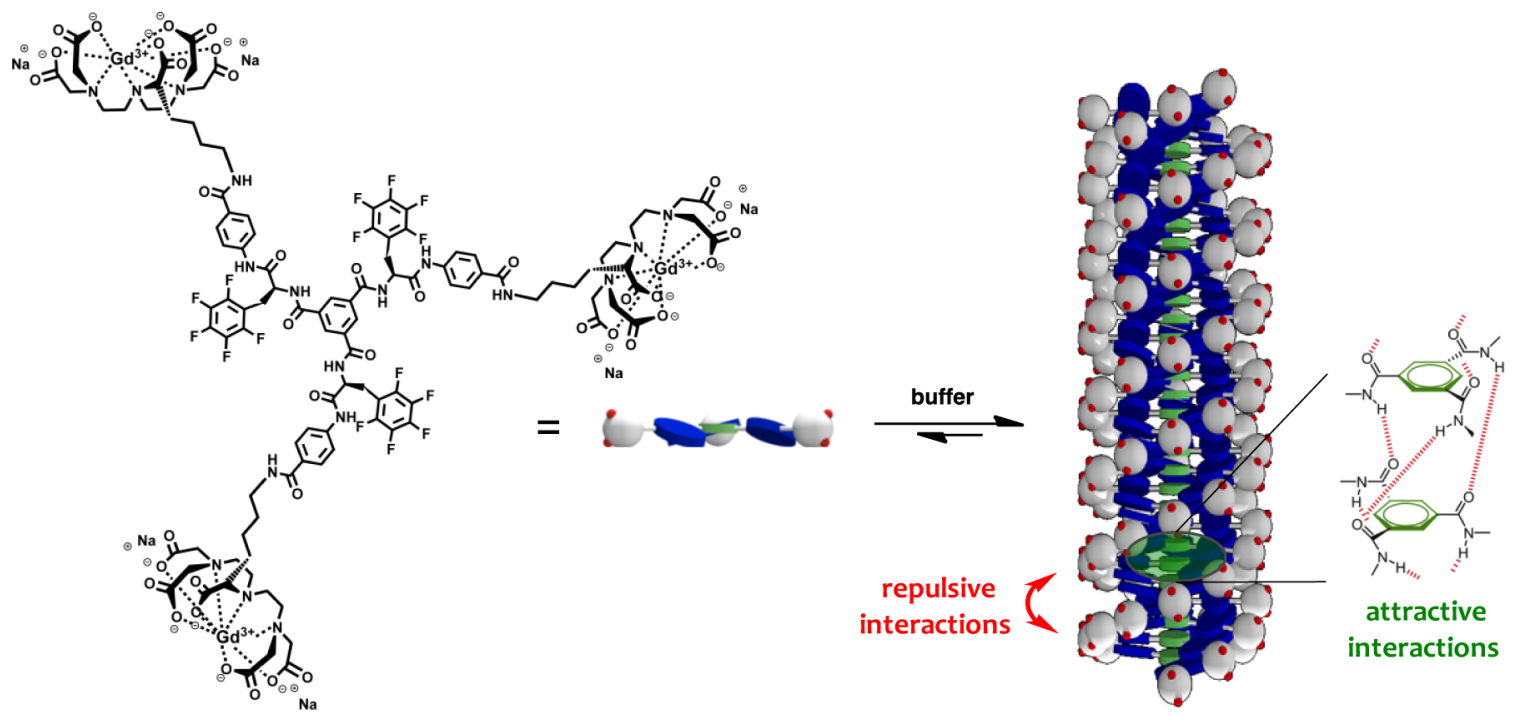

Figure 1. Molecular structure of the $C_{3}$-symmetrical discotic amphiphile $\left(C_{3} D A\right)$ and the schematic representation of the self-assembly into a helically ordered architecture.

constant of the supramolecular polymerization, which is a direct measure for the degree of cooperativity involved. The latter can be adjusted with the ionic strength, opening up opportunities to control the size of the self-assembled macromolecules. Furthermore, we apply a statistical thermodynamic theory describing the temperature-dependent supramolecular polymerization of charged species in water, allowing us to extract quantitative information on the effective surface charge densitiy $\nu$ of the $\mathrm{C}_{3} \mathrm{DA}$ assemblies. The charge density is a key property that has been shown to determine the shape and size of supramolecular polymers, ${ }^{8 c, d, 10}$ the long-range crystallization of self-assembling filaments ${ }^{11}$ and the bioactivity of self-assembled biomaterials. ${ }^{12}$ Because charge densities are notoriously difficult to determine experimentally in self-assembled systems, ${ }^{13}$ their quantification and the ability to tune their magnitude, are highly desirable.

Variable-temperature CD spectroscopy has in the past been shown to be a sensitive tool to monitor the growth of helical supramolecular polymers and oligomers. ${ }^{6 \mathrm{~b}, \mathrm{~d}, 8 \mathrm{~d}, \mathrm{f}, 10 \mathrm{a}}$ Thermodynamic parameters characteristic for the supramolecuar polymerization process can be extracted by applying a nucleated selfassembly model. ${ }^{6}$ Values for the polymerization temperature $T_{\mathrm{p}}$ and the enthalpy of elongation $\Delta H_{\mathrm{e}}$, as well as the degree of cooperativity expressed in a dimensionless activation constant $K_{a}$ are accurately determined by fitting temperature-dependent circular dichroism (CD) spectroscopic measurements with this model. The activation constant $K_{a}$ is unity for isodesmic processes and very much smaller than that for cooperative ones. By performing $C D$ measurements as a function of the temperature $T$ and ionic strength $I$ of the medium, that is, by varying the $\mathrm{NaCl}$ concentration, we can assess how the thermodynamic parameters relating to the self-assembly behavior of $\mathrm{C}_{3} \mathrm{DAs}$ in aqueous solution vary as a function of the salinity. The theory that we invoke to analyze and rationalize how $T_{\mathrm{p}}, \Delta H_{\mathrm{e}}$, and $K_{\mathrm{a}}$ change with ionic strength, is based on a combination of a mass action model for linear selfassembly (via a nucleation-elongation mechanism) ${ }^{6}$ and a model that describes the ionic strength dependence of the electrostatic free energy of relatively weakly charged, quasi onedimensional objects (within the Debye-Hückel approximation). ${ }^{14}$ The details of the calculations and model assumptions, as well as a justification of these, can be found in the Supporting Information (SI). We first investigate the $\mathrm{CD}$ spectra of charged $\mathrm{C}_{3} \mathrm{DA}$ at room temperature, in which repulsive electrostatic interactions act against the cooperative and nucleated self-assembly mechanism that is coded into their triple hydrogen bonding hydrophobic core (Figure 1). By increasing the ionic strength $I$ via the addition of $\mathrm{NaCl}$ into the PBS buffer, CD spectra at room temperature show a shift in the negative band from $262 \mathrm{~nm}(I=0.17 \mathrm{M})$ to $278 \mathrm{~nm}(I=1 \mathrm{M}$; Figure 2A). At high ionic strength, the bisignate Cotton effect for self-assembled charged $\mathrm{C}_{3} \mathrm{DAs}$ has the same shape as discotics equipped with a charge neutral peripheral complex. ${ }^{8 \mathrm{~d}}$ This similarity between the Cotton effects indicates that repulsive interactions at the periphery of the nanorods are weakened and the packing of the core aromatic chromophores in the helical aggregates changes accordingly.

The temperature-dependent $\mathrm{CD}$ cooling curves of $\mathrm{C}_{3} \mathrm{DA}$ in PBS buffer at various ionic strengths show pronounced changes in their shape (Figure 2B). Fitting the profiles at two different discotic concentrations $\left(6 \times 10^{-6} \mathrm{M}\right.$ and $\left.8 \times 10^{-6} \mathrm{M}\right)$ with the nucleation-elongation self-assembly model $^{6 a, c}$ produces values for $T_{\mathrm{p}}, \Delta H_{\mathrm{e}}$, and $K_{\mathrm{a}}$ (see SI for full details and Tables $\mathrm{S} 1$ and S2). In agreement with the theory, the polymerization temperature $T_{\mathrm{p}}$ (the critical temperature below which elongation becomes thermodynamically favorable) shifts to higher temperatures with increasing ionic strength [Figure S3A, eq (46) in the SI]. We also find that the enthalpy of elongation $\Delta H_{\mathrm{e}}$ increases with ionic strength, as expected from the theory [Figure S3B, eq (51) in the SI].

Crucially, we were intrigued to observe that the dimensionless activation constant $K_{\mathrm{a}}$ decreases upon increasing the ionic strength, indicative for a more cooperative self-assembly process. This is a direct result of a change in the self-assembly mechanism, as previously hypothesized, from a frustrated growth yet near-isodesmic mechanism (with $K_{\mathrm{a}} \approx 10^{-1}$ ) to a strongly cooperative system (with $K_{\mathrm{a}} \approx 10^{-4}$ ). Importantly, adding a water-soluble nonionic organic crowding agent, $\mathrm{L}^{-}$ mannitol, instead of $\mathrm{NaCl}$ did not lead to significant changes in the $\mathrm{CD}$ shape or cooling curve of the self-assembled helical $\mathrm{C}_{3} \mathrm{DAs}$ (Figure S5). Excluded-volume effects at these low selfassembling $\mathrm{C}_{3} \mathrm{DA}$ concentrations can therefore be neglected. 

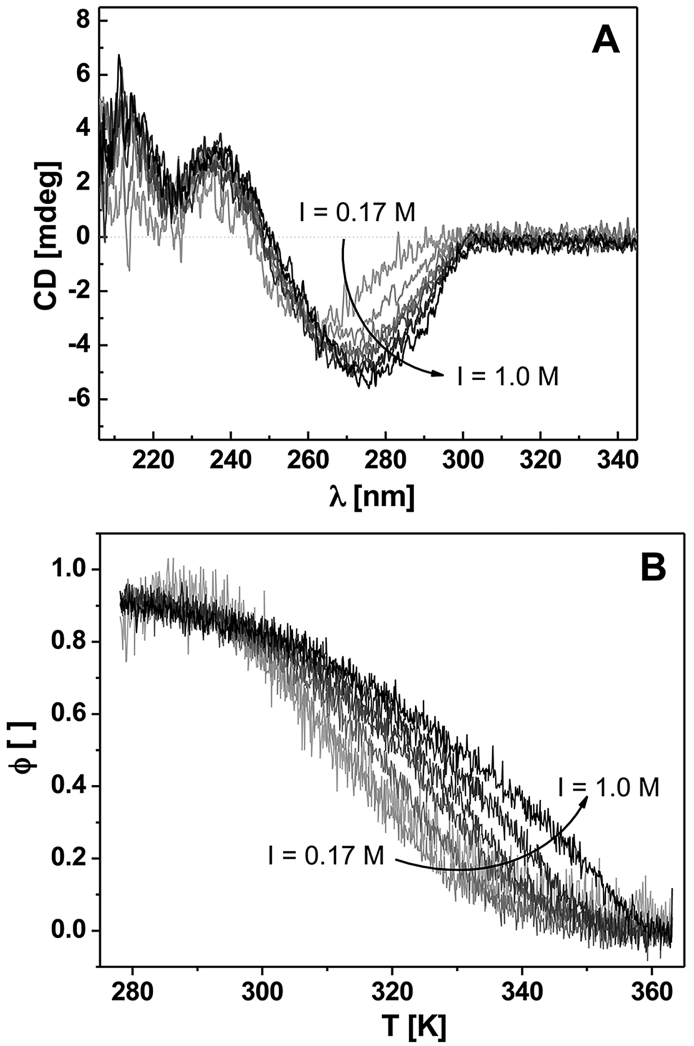

Figure 2. (A) Ionic strength $I$ dependent room temperature $C D$ spectra for the Gd(III)-DTPA discotic amphiphile and (B) the corresponding cooling curves from the molecularly dissolved state [PBS buffer (10 mM, pH 7.4), $I=0.17-1.0 \mathrm{M}$ ], monitored at $\lambda=270$ $\mathrm{nm}$; the normalized data is shown as degree of aggregation $\phi$ versus temperature T: 0 referring to the molecular dissolved state and 1 to a fully polymerized system; in all cases, the discotic concentration was kept constant at $8 \times 10^{-6} \mathrm{M}$.

According to our model calculations for the nucleated selfassembly of linear charged objects in water, $K_{\mathrm{a}}$ scales with the ionic strength $I$ (in $\mathrm{m}^{-3}$ ) as follows [eq (27), SI]: $\ln K_{\mathrm{a}}(I)=$ $\ln K_{\mathrm{a}}(\infty)+\nu^{2} \lambda_{\mathrm{D}} \lambda_{\mathrm{B}}$, where $\nu$ is the projected linear charge density, that is, the number of charges per unit length, $\lambda_{\mathrm{B}}$, the Bjerrum length $[=0.71 \mathrm{~nm}$ at room temperature, see SI, eq (10)], $\lambda_{\mathrm{D}}=1 /\left(8 \pi \lambda_{\mathrm{B}} I\right)^{1 / 2}$, the Debye screening length, and $I$, the number density of monovalent salt (dimension $\mathrm{m}^{-3}$ ). So, adding salt makes the assembly more cooperative, which indeed is what we observe experimentally. By fitting $\ln K_{\mathrm{a}}(I)$ to the predicted $1 / \sqrt{ } I$ dependence in Figure 3 , we obtain values for the scale factor $\nu^{2}\left(\lambda_{\mathrm{B}} / 8 \pi\right)^{1 / 2} \approx 1 \times 10^{14} \pm 0.9 \times 10^{13}$, allowing us to extract the (effective) linear charge density $\nu$ of the nanorods in aqueous solution equivalent to $4.5 \pm 0.3 \mathrm{~nm}^{-1}$.

Obviously, this value is merely a projected quantity, since we are dealing with self-assembled cylindrical (rod-like) objects with a well-defined diameter that bear negative charges at their surface. Knowing the diameter of the cylinders of $6 \mathrm{~nm}$ (width of the molecular building blocks), ${ }^{8 \mathrm{~d}}$ we can calculate their surface area per $\mathrm{nm}\left(\pi \times 6 \mathrm{~nm} \times 1 \mathrm{~nm}=18.9 \mathrm{~nm}^{2}\right)$. Based on this, we can extrapolate the effective linear charge density values into effective surface charge densities of $0.24 \pm 0.02 \mathrm{~nm}^{-2}$. The found value is lower than the expected one of $0.91 \mathrm{~nm}^{-2}$, calculated using a cylindrical surface area per $\mathrm{nm}$ of $18.9 \mathrm{~nm}^{2}$, by approximating the $\mathrm{C}_{3} \mathrm{DA}-\mathrm{C}_{3} \mathrm{DA}$ distance ${ }^{15}$ in the selfassembled stacks to be $0.35 \mathrm{~nm}$ and by assuming that $\mathrm{C}_{3} \mathrm{DA}$

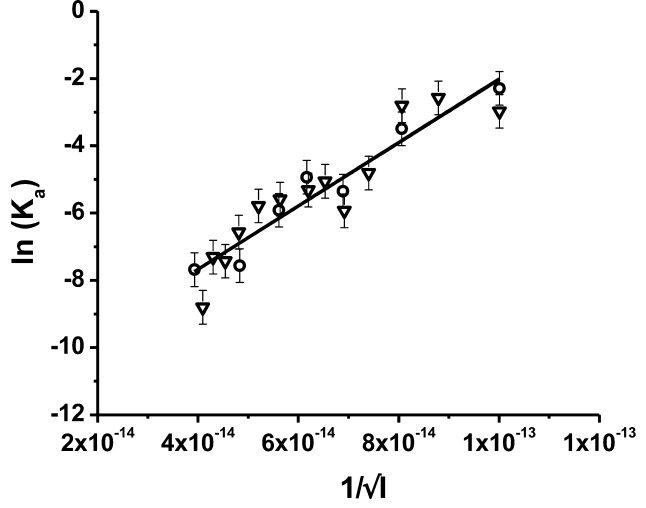

Figure 3. Degree of cooperativity $K_{\mathrm{a}}$ as a function of the ionic strength $I$ in the self-assembly of ionic discotic amphiphiles [concentration of 6 $\times 10^{-6} \mathrm{M}$ (triangles) and $8 \times 10^{-6} \mathrm{M}$ (circles)]. The graph is plotted as $\ln \left(K_{\mathrm{a}}\right)$ vs $1 / \sqrt{ } I$, with $I$ expressed as $\mathrm{m}^{-3}$, as predicted theoretically: see the SI. The linear fit of the data points $\left[R^{2}=0.86\right.$ (triangles) and 0.94 (circles)] gives as slope $1 \times 10^{14} \pm 0.9 \times 10^{13}$ for both concentrations of the discotic amphiphile.

carry the maximum of 6 fully ionized charges. In comparison, the surface charge density of double helical DNA is $\sim 1 \mathrm{~nm}^{-2}, 16$ which is considered to be a particularly strongly charged polyelectrolyte.

We attribute the discrepancy between the nominal and found surface charge density to counterion condensation, that is, counterions that are effectively bound to the highly charged surfaces of the self-assembled nanostructures. ${ }^{17}$ We do not find any effects of nonuniform counterion condensation, although this is not surprising given that the relevant conditions, such as a large Debye screening length on the scale of the width of the assemblies, are not met. ${ }^{18}$ While describing a nominally highly charged cylindrical self-assembly by a line charge, as we have done, and applying an electrostatic theory valid for low charge densities, may appear an oversimplification, the theory does predict effectively the impact that ionic strength has on the thermodynamic parameters describing $\mathrm{C}_{3} \mathrm{DA}$ self-assembly. The trends of $T_{\mathrm{p}}, \Delta H_{\mathrm{e}}$, and $K_{\mathrm{a}}$ with increasing ionic strength predicted by the model agree well with the experimental findings. Moreover, it confirms our observation that screening of the Coulomb interactions between charges on individual assemblies by mobile ions enhances the cooperativity of the equilibrium polymerization.

It is important to note that effective charge densities in related supramolecular systems have been particularly difficult to assess experimentally, ${ }^{8 \mathrm{~b}-\mathrm{d}, 10-12}$ which is remarkable because subtle changes in the molecular structure of the ionic monomers have been shown to lead to dramatic effects in the self-assembly properties and function of the obtained materials. Early key studies on balancing attractive and repulsive interactions in aqueous self-assembly include the work of van Esch et al. $^{8 \mathrm{~b}}$ on cyclohexane-amino acid conjugates, which form responsive hydrogels that can be reversibly switched from gel to sol by changing the $\mathrm{pH}$. Another important study was reported by Hartgerink et al. ${ }^{8 \mathrm{c}}$ using multidomain peptides to control the self-assembly of nanostructured fibers. In both systems, Coulombic repulsive interactions introduced via charged amino acid moieties were the primary reason for disassembly to occur. In line with these studies and our own previous investigations, ${ }^{8 \mathrm{~d}, \mathrm{f}, 19}$ our findings hereby confirm once 
more that balancing attractive and repulsive interactions allows the control of the growth of ordered supramolecular polymers.

In conclusion, we put forward a model for the nucleated linear self-assembly of charged species in order to analyze how salt alters the reversible polymerization of $\mathrm{C}_{3} \mathrm{DA}$ in water. The model confirms our experimental observation that screening of repulsive Coulombic interactions by added salt leads to a significant increase in the cooperativity of the polymerization. The findings we present here are significant: while it was known that screening of interactions between charges enhance elongation, ${ }^{14 \mathrm{c}}$ the impact it has on the degree of cooperativity in the equilibrium polymerization was not known. Because cooperativity is crucial to influencing the length and shape of the supramolecular nanorods in solution, accessible and tunable ways to control cooperativity are highly important. Our approach is generally applicable to quasi one-dimensional supramolecular polymerizations in aqueous solution or in the gel-state and particularly relevant for biomedical applications ranging from tissue engineering and drug delivery to molecular imaging.

\section{ASSOCIATED CONTENT}

\section{S Supporting Information}

Experimental details and materials, fitting procedures and extraction of thermodynamic parameters as a function of the ionic strength; additional experimental data (CD spectra and cooling curves) and derivation of the model. This material is available free of charge via the Internet at http://pubs.acs.org.

\section{AUTHOR INFORMATION}

\section{Corresponding Author}

*E-mail: p.vanderschoot@phys.tue.nl; p.besenius@unimuenster.de.

\section{Notes}

The authors declare no competing financial interest.

\section{ACKNOWLEDGMENTS}

We thank Marko Nieuwenhuizen and Dr. Tom de Greef for helpful discussions. We acknowledge IBOS (Project 053.63.310), Marie Curie Actions FP7 (SahnMat, PIEF-GA2009-235914) [P.B.], and The Netherlands Organization for Scientific Research (NWO VENI Grant 700.10.406) [I.K.V.] for funding.

\section{REFERENCES}

(1) Kyrylyuk, A. V.; Hermant, M. C.; Schilling, T.; Klumperman, B.; Koning, C. E.; van der Schoot, P. Nat. Nanotechnol. 2011, 6, 364-369.

(2) Mömming, C. M.; Kehr, G.; Wibbeling, B.; Fröhlich, R.; Schirmer, B.; Grimme, S.; Erker, G. Angew. Chem., Int. Ed. 2010, 49, 2414-2417.

(3) Tielrooij, K. J.; Garcia-Araez, N.; Bonn, M.; Bakker, H. J. Science 2010, 328, 1006-1009.

(4) (a) Hunter, C. A.z; Anderson, H. L. Angew. Chem., Int. Ed. 2009, 48, 7488-7499. (b) Ercolani, G.; Schiaffino, L. Angew. Chem., Int. Ed. 2011, 50, 2-9.

(5) Whitty, A. Nat. Chem. Biol. 2008, 4, 435-439.

(6) (a) van der Schoot, P. In Supramolecular Polymers, 2nd ed.; Ciferri, A., Ed.; Taylor \& Francis: London, 2005; (b) Jonkheijm, P.; van der Schoot, P.; Schenning, A. P. H. J.; Meijer, E. W. Science 2006, 313, 80-83. (c) de Greef, T. F. A.; Smulders, M. M. J.; Wolffs, M.; Schenning, A. P. H. J.; Sijbesma, R. P.; Meijer, E. W. Chem. Rev. 2009, 109, 5687-5754. (d) Helmich, F.; Lee, C. C.; Nieuwenhuizen, M. M. L.; Gielen, J. C.; Christianen, P. C. M.; Larsen, A.; Fytas, G.; Leclère, P.
E. L. G.; Schenning, A. P. H. J.; Meijer, E. W. Angew. Chem., Int. Ed. 2010, 49, 3939-3942.

(7) Filot, I. A. W.; Palmans, A. R. A.; Hilbers, P. A. J.; van Santen, R. A.; Pidko, E. A.; de Greef, T. F. A. J. Phys. Chem. B 2010, 114, 1366713674.

(8) (a) Stupp, S. I.; LeBonheur, V.; Walker, K.; Li, L. S.; Huggins, K. E.; Keser, M.; Amstutz, A. Science 1997, 276, 384-389. (b) van Bommel, K. J. C.; van der Pol, C.; Muizebelt, I.; Friggeri, A.; Heeres, A.; Meetsma, A.; Feringa, B. L.; van Esch, J. Angew. Chem., Int. Ed. 2004, 43, 1663-1667. (c) Dong, H.; Paramonov, S. E.; Aulisa, L.; Bakota, E. L.; Hartgerink, J. D. J. Am. Chem. Soc. 2007, 129, 1246812472. (d) Besenius, P.; Portale, G.; Bomans, P. H. H.; Janssen, H. M.; Palmans, A. R. A.; Meijer, E. W. Proc. Natl. Acad. Sci. U.S.A. 2010, 107, 17888-17893. (e) Roman, M.; Cannizzo, C.; Pinault, T.; Isare, B.; Andrioletti, B.; van der Schoot, P.; Bouteiller, L. J. Am. Chem. Soc. 2010, 132, 16818-16824. (f) Besenius, P.; van den Hout, K. P.; Albers, H. M. H. G.; Greef, T. F. A.; Olijve, L. L. C.; Hermans, T. M.; de Waal, B. F. M.; Bomans, P. H. H.; Sommerdijk, N. A. J. M.; Portale, G.; Palmans, A. R. A.; van Genderen, M. H. P.; Vekemans, J. A. J. M.; Meijer, E. W. Chem.-Eur. J. 2011, 17, 5193-5203. (g) Boekhoven, J.; Brizard, A. M.; van Rijn, P.; Stuart, M. C. A.; Eelkema, R.; van Esch, J. H. Angew. Chem., Int. Ed. 2011, 50, 12285-12289.

(9) (a) Imae, T.; Ikeda, S. Colloid Polym. Sci. 1987, 265, 1090-1098. (b) MacKintosh, F. C.; A.Safran, S.; Pincus, P. A. Europhys. Lett. 1990, 12, 697-702. (c) Safran, S. A.; Pincus, P. A.; Cates, M. E.; MacKintosh, F. C. J. Phys. (Paris) 1990, 51, 503-510. (d) May, S.; A. Ben-Shaul, M. J. Phys. Chem. B 2001, 105, 630-640.

(10) (a) Ghosh, A.; Haverick, M.; Stump, K.; Yang, X.; Tweedle, M. F.; Goldberger, J. E. J. Am. Chem. Soc. 2012, 134, 3647-3650. (b) Besenius, P.; Heynens, J. L. M.; Straathof, R; Nieuwenhuizen, M. M. L.; Bomans, P. H. H.; Terreno, E.; Aime, S.; Strijkers, G. J.; Nicolay, K.; Meijer, E. W. Contrast Media Mol. Imaging 2012, 7, 356361.

(11) Cui, H.; Pashuck, E. T.; Velichko, Y. S.; Weigand, S. J.; Cheetham, A. G.; Newcomb, C. J.; Stupp, S. I. Science 2009, 327, 555559.

(12) Goldberger, J. E.; Berns, E. J.; Bitton, R.; Newcomb, C. J.; Stupp, S. I. Angew. Chem., Int. Ed. 2011, 50, 6292-6295.

(13) (a) Bordi, F.; Cametti, C.; Tan, J. S.; Boris, D. C.; Krause, W. E.; Plucktaveesak, N.; Colby, R. H. Macromolecules 2002, 35, 7031-7038. (b) Böhme, U.; Scheler, U. Adv. Colloid Interface Sci. 2010, 158, 6367.

(14) (a) Odijk, T. J. Phys. Chem. 1989, 93, 3888-3889. (b) Odijk, T. J. Chem. Phys. 1990, 93, 5172-5176. (c) Odijk, T. Biophys. Chem. 1991, 41, 23-29. (d) Duyndam, A.; Odijk, T. Langmuir 1996, 12, $4718-4722$

(15) Stals, P. J. M.; Smulders, M. M. J.; Martin-Rapun, R.; Palmans, A. R. A.; Meijer, E. W. Chem.-Eur. J. 2009, 15, 2071-2080.

(16) Anderson, C. F.; Record, M. T. Annu. Rev. Phys. Chem. 1982, 33, $191-222$.

(17) (a) Manning, G. S. J. Chem. Phys. 1969, 51, 924-933. (b) Deserno, M.; Holm, C.; May, S. Macromolecules 1999, 33, 199206.

(18) Odijk, T. Phys. A 1991, 176, 201-205.

(19) Kegel, W. K.; van der Schoot, P. Biophys. J. 2004, 86, 39053913. 